\title{
Processed meat consumption and lung health: more evidence for harm
}

\author{
Raphaëlle Varraso ${ }^{1,2}$ and Carlos A. Camargo $\mathrm{Jr}^{3}$
}

\begin{abstract}
Affiliations: 'INSERM, CESP Centre for Research in Epidemiology and Population Health, U1018, Respiratory and Environmental Epidemiology Team, Villejuif, and ${ }^{2}$ Univ Paris Sud 11, UMRS 1018, Villejuif, France. ${ }^{3}$ Dept of Emergency Medicine, Massachusetts General Hospital, Harvard Medical School, Boston, MA, USA.
\end{abstract}

Correspondence: R. Varraso, INSERM, CESP Centre for Research in Epidemiology and Population Health, U1018, Respiratory and Environmental Epidemiology Team, 16 Avenue Paul Vaillant-Couturier, F-94807 Villejuif Cedex, France. E-mail: raphaelle.varrasodinserm.fr

@ERSpublications

Data suggest processed meat intake may adversely affect lung health and the risk of COPD http:// ow.ly/tgh 81

In the recent Global Burden of Disease study [1], chronic obstructive pulmonary disease (COPD) was the third most common cause of death worldwide in 2010. The predominant risk factor for COPD in the developed world is cigarette smoking, but up to one-third of COPD patients have never smoked, suggesting that other factors are involved. Relatively little attention has been paid to these other factors, such as diet, and how their modification might decrease the global burden of COPD. Nevertheless, the lungs exist in a high-oxygen environment and it is reasonable to posit that certain exposures (and local inflammation) can increase the burden of oxidants further. The balance between these potentially toxic substances and the protective actions of antioxidant defences, including those derived from diet, may play a role in the loss of lung function over time and the eventual development of COPD.

Based on this prevailing hypothesis, most of the diet-lung research to date has focused on dietary factors with antioxidant or anti-inflammatory properties, including: vitamin $\mathrm{E}$ (present in seed oils and nuts); vitamin C (fruits and vegetables); vitamin A, provitamin A carotenoids, and lycopene (fruits and vegetables); and selenium (seeds, seafood, and fish) [2]. In addition to these nutrients with antioxidant properties, the lipid composition of the diet (e.g. omega-3 fatty acids) and vitamin D also have been studied for their potential role in lung health $[2,3]$. Although most of the "promising" results for these nutrients have been from cross-sectional studies, a few longitudinal studies exist that demonstrate favourable associations between specific dietary factors and a slower decline of lung function or lower incidence of COPD. All of this nutrient-oriented research fails, in some respects, to appreciate the complexity of dietary assessment. For example, due to the fact we eat meals instead of isolated nutrients or even foods, several authors have proposed assessing dietary patterns to gain a broader picture of diet [4]. Dietary patterns might be seen as offering a more "agnostic" approach (similar to genome-wide association studies), whereas nutrients/foods could be seen as more of a hypothesis-driven, pathway-based approach. Either way, recent studies of dietary patterns also have shown associations with lung health. Out of five studies looking at the association between dietary patterns with spirometry or COPD symptoms or incidence [5-9], three studies reported a "protective" association for a "prudent" dietary pattern characterised by a high intake of fruit, vegetables, fish and wholegrain cereals $[5,8,9]$, consistent with the dietary antioxidant or antiinflammatory properties hypothesis. Moreover, four studies (out of the five) also reported a deleterious effect of a "Western" diet characterised by a high intake of chicken, pork, fish, rice and noodle dishes, and preserved foods among a population of Chinese Singaporeans [7], by a high intake of cured and red meat, potato, boiled vegetables, added fat, coffee and beer among Dutch adults [6], and by a high intake of cured

Received: Dec 302013 | Accepted: Jan 162014

Conflict of interest: None declared.

Copyright @ERS 2014 
and red meats, refined grains, desserts, sweets, French fries and high-fat dairy products among adults in the USA $[8,9]$.

In this issue of the European Respiratory Journal, Окиво et al. [10] report diet-lung results from a crosssectional analysis of 1551 males and 1391 females from the UK. Briefly, they found that consumption of processed meats (i.e. bacon, gammon, ham, corned beef, spam and luncheon meat, sausage, and meat pies) was associated with worse lung function, in both males and females. These results are consistent with a previous cross-sectional study in a large US population showing that frequent consumption of cured meats was associated with lower forced expiratory volume in $1 \mathrm{~s}$ (FEV1) and FEV1/forced vital capacity and an increased risk of COPD [11], and with two longitudinal studies of male [12] and female [13] health professionals where we reported that cured meat intake was positively associated with the risk of newly diagnosed COPD. More recently, a longitudinal study of COPD patients in Spain showed that frequent consumption of cured meats was associated with more COPD exacerbations [14]. Aside from lung health, recent studies in the USA [15] and Europe [16] reported that a high consumption of processed meat was associated with higher risk of coronary heart diseases, diabetes mellitus and all-cause mortality [17]. Likewise, cancer prevention guidelines recommend avoidance of processed meat [18], even if researchers have not yet been able to determine the key factor linking processed meat and cancer.

One possible mechanism for the association between processed meat intake, or more precisely, "cured meat" intake, and the risk of COPD is related to the nitrites added to meat products as preservatives and colour fixatives [19]. Based on the processing technologies, processed meat products that are treated with small amounts of nitrite, either as dry salt or as salt solution in water, are classified as cured meat. Dietary nitrites may generate reactive nitrogen species that can amplify inflammatory processes in the airways and lung parenchyma causing DNA damage, inhibition of mitochondrial respiration, and nitrosative stress. The long-term persistence of nitrosative stress may contribute to the progressive deterioration of pulmonary function and may be implicated in the pathogenesis of COPD [20]. Animal studies have reported that rats drinking water enriched with sodium nitrite for 2 years developed pulmonary emphysema [21]. However, the role of dietary nitrites and nitrates in health is matter of debate and consideration of food sources of nitrates and nitrites as healthful dietary components has received recent attention [22, 23], especially in cardiovascular diseases [24].

Another potential mechanism suggested by the authors is related to the advanced glycation end-products (AGEs), which occur naturally in meat and are formed through heat processing, and may also have proinflammatory actions [25]. In the diabetes field, excessive consumption of AGEs with the standard Western diet are increasingly seen as a potential risk for insulin resistance and for disease itself [25]. Apart from these two mechanisms mentioned by the authors, inflammatory biomarkers might also be a link between processed meat intake and lung health. Positive associations have been observed between processed meat and pro-inflammatory biomarkers [26], which in turn have been associated with a higher risk of COPD and COPD exacerbations [27]. To clarify the role of processed meat in the physiopathology of respiratory diseases, more mechanistic studies are needed (e.g. prospective studies with biomarkers of nitrosative stress and inflammation).

As the authors recognise, the novelty of their article rests largely on their evaluation of effect modification by other sources of antioxidants (dietary sources) and oxidants (smoking) on the association between processed meat and lung function. When the analysis was stratified according to the level of antioxidants (fruit and vegetable intake, but also a dietary score reflecting the total antioxidant capacity of diet, the total antioxidant capacity (TAC) score), the deleterious association of processed meat on lung function was reported only in males with the lower dietary intake. By contrast, when the analysis was stratified according to the level of oxidants (smoking), the negative association between processed meat intake and lung function was more marked among male current smokers. We think that this gender difference deserves further study, as food choices are an area in which research has revealed consistent behavioural gender differences. Food choice is dependent on a wide spectrum of factors, which affect human behaviour in different ways, resulting in the choice of some specific products and in the rejection of others [28]. Studies conducted in modern Western societies report consistent associations between gender and specific foods, where meat (especially red and processed meat), alcohol and hearty portion sizes are associated with masculinity, while vegetables, fruit, fish and sour dairy products (e.g. yogurt and cottage cheese) are associated with femininity [29]. Thus, it is possible that females just do not eat enough processed meats and, therefore, do not reach the threshold where lung effects might be seen. It is also possible that the harmful effect of processed meat is dependent on the initial level of antioxidants (here fruits and vegetables), explaining why the hypothesis of food balance is seen in males only (who reported a lower intake of fruits and vegetables as compared to females). Besides these gender differences in dietary intakes, there was also a 
gender difference regarding smoking patterns, with roughly two-thirds of males being ever-smokers as compared to only one-third of females.

Besides diet per se, physical activity and body mass index (BMI) also have been suggested as aetiological factors for lung function decline or onset of COPD. Regular physical activity may reduce lung function decline and risk of developing COPD among active smokers [30]. Low body weight, as indicated by BMI, has been reported as an important risk factor for subsequent development of COPD in males [31]. Of course, each of these nutritional factors (diet, body composition, and physical activity) are intrinsically linked to one another, for example, body composition is the result of both dietary behaviour and physical activity (and also genetic factors). Future research will need to elucidate how nutritional factors interact in relation to lung health and, more specifically, to the risk of COPD.

In the meantime, prior publications [11-14] and the new results of OKUво et al. [10] suggest that processed meat intake may adversely affect lung function and the risk of COPD. Although further longitudinal studies would help to clarify the nature of this association, we anticipate that well-designed, randomised trials will be needed to settle the causality of the observed epidemiological associations. From a public health perspective, stopping smoking is still more important than cutting down intake of processed meat, but, based on the current evidence, we encourage moderation (or complete avoidance) of processed meat intake, as already recommended by several national dietary guidelines [32].

\section{References}

1 Lozano R, Naghavi M, Foreman K, et al. Global and regional mortality from 235 causes of death for 20 age groups in 1990 and 2010: a systematic analysis for the Global Burden of Disease Study 2010. Lancet 2012; 380: 2095-2128.

2 Hanson C, Rutten EPA, Wouters EFM, et al. Diet and vitamin D as risk factors for lung impairment and COPD. Transl Res 2013; 162: 219-236.

3 Calder PC. n-3 polyunsaturated fatty acids, inflammation, and inflammatory diseases. Am J Clin Nutr 2006; 83: Suppl. 6, 1505S-1519S.

$4 \quad$ Hu FB. Dietary pattern analysis: a new direction in nutritional epidemiology. Curr Opin Lipidol 2002; 13: 3-9.

5 Shaheen SO, Jameson KA, Syddall HE, et al. The relationship of dietary patterns with adult lung function and COPD. Eur Respir J 2010; 36: 277-284.

6 McKeever TM, Lewis SA, Cassano PA, et al. Patterns of dietary intake and relation to respiratory disease, forced expiratory volume in $1 \mathrm{~s}$, and decline in 5-y forced expiratory volume. Am J Clin Nutr 2010; 92: 408-415.

7 Butler LM, Koh W-P, Lee H-P, et al. Prospective study of dietary patterns and persistent cough with phlegm among Chinese Singaporeans. Am J Respir Crit Care Med 2006; 173: 264-270.

8 Varraso R, Fung TT, Barr RG, et al. Prospective study of dietary patterns and chronic obstructive pulmonary disease among US women. Am J Clin Nutr 2007; 86: 488-495.

9 Varraso R, Fung TT, Hu FB, et al. Prospective study of dietary patterns and chronic obstructive pulmonary disease among US men. Thorax 2007; 62: 786-791.

10 Okubo H, Shaheen SO, Ntani G, et al. Processed meat consumption and lung function: modification by antioxidants and smoking. Eur Respir J 2014; 43: 972-982.

11 Jiang R, Paik DC, Hankinson JL, et al. Cured meat consumption, lung function, and chronic obstructive pulmonary disease among United States adults. Am J Respir Crit Care Med 2007; 175: 798-804.

12 Varraso R, Jiang R, Barr RG, et al. Prospective study of cured meats consumption and risk of chronic obstructive pulmonary disease in men. Am J Epidemiol 2007; 166: 1438-1445.

13 Jiang R, Camargo CA Jr, Varraso R, et al. Consumption of cured meats and prospective risk of chronic obstructive pulmonary disease in women. Am J Clin Nutr 2008; 87: 1002-1008.

14 de Batlle J, Mendez M, Romieu I, et al. Cured meat consumption increases risk of readmission in COPD patients. Eur Respir J 2012; 40: 555-560.

15 Pan A, Sun Q, Bernstein AM, et al. Red meat consumption and mortality: results from 2 prospective cohort studies. Arch Intern Med 2012; 172: 555-563.

16 Rohrmann S, Overvad K, Bueno-de-Mesquita HB, et al. Meat consumption and mortality - results from the European Prospective Investigation into Cancer and Nutrition. BMC Med 2013; 11: 63.

17 Micha R, Wallace SK, Mozaffarian D. Red and processed meat consumption and risk of incident coronary heart disease, stroke, and diabetes mellitus: a systematic review and meta-analysis. Circulation 2010; 121: 2271-2283.

18 World Cancer Research Fund, American Institute for Cancer Research. Food, Nutrition, Physical Activity, and the Prevention of Cancer: a Global Perspective. Washington, American Institute of Cancer Research, 2007.

19 Jakszyn P, Agudo A, Ibáñez R, et al. Development of a food database of nitrosamines, heterocyclic amines, and polycyclic aromatic hydrocarbons. J Nutr 2004; 134: 2011-2014.

20 Ricciardolo FLM, Di Stefano A, Sabatini F, et al. Reactive nitrogen species in the respiratory tract. Eur J Pharmacol 2006; 533: 240-252.

21 Shuval HI, Gruener N. Epidemiological and toxicological aspects of nitrates and nitrites in the environment. Am J Public Health 1972; 62: 1045-1052.

22 Hord NG, Tang Y, Bryan NS. Food sources of nitrates and nitrites: the physiologic context for potential health benefits. Am J Clin Nutr 2009; 90: 1-10.

23 Cunningham E. Dietary nitrates and nitrites-harmful? Helpful? Or paradox? J Acad Nutr Diet 2013; 113: 1268.

24 Machha A, Schechter AN. Dietary nitrite and nitrate: a review of potential mechanisms of cardiovascular benefits. Eur J Nutr 2011; 50: 293-303.

25 Vlassara H, Uribarri J. Advanced glycation end products (AGE) and diabetes: cause, effect, or both? Curr Diab Rep 2014; 14: 453. 
Ley SH, Sun Q, Willett WC, et al. Associations between red meat intake and biomarkers of inflammation and glucose metabolism in women. Am J Clin Nutr 2014; 99: 352-360.

27 Thomsen M, Ingebrigtsen TS, Marott JL, et al. Inflammatory biomarkers and exacerbations in chronic obstructive pulmonary disease. JAMA 2013; 309: 2353-2361.

28 Arganini C, Saba A, Comitato R, et al. Gender differences in food choice and dietary intake in modern Western societies. In: Maddock J, ed. Public Health - Social and Behavioral Health. Rijeka, InTechOpen, 2012; pp. 83-102.

29 O’Doherty Jensen K, Holm L. Preferences, quantities and concerns: socio-cultural perspectives on the gendered consumption of foods. Eur J Clin Nutr 1999; 53: 351-359.

30 Garcia-Aymerich J, Lange P, Benet M, et al. Regular physical activity modifies smoking-related lung function decline and reduces risk of chronic obstructive pulmonary disease: a population-based cohort study. Am J Respir Crit Care Med 2007; 175: 458-463.

31 Harik-Khan RI, Fleg JL, Wise RA. Body mass index and the risk of COPD. Chest 2002; 121: 370-376.

32 Willett WC, Stampfer MJ. Current evidence on healthy eating. Annu Rev Public Health 2013; 34: 77-95. 